\title{
EL MÉTODO PROYECTO EN LA EDUCACIÓN AMBIENTAL DESDE LAS CLASES DE INGLÉS: RETO EN LA FORMACIÓN UNIVERSITARIA.
}

\author{
THE PROJECT METHOD IN ENVIRONMENTAL EDUCATION FROM \\ THE ENGLISH CLASSES: A CHALLENGE IN UNIVERSITY TRAINING
}

\author{
Fernando Emilio Valladares Fuente \\ Luis Enrique Hernández Amaro \\ luisenrique.6611@yahoo.com
}

\section{RESUMEN}

El método proyectos en la formación de los estudiantes universitarios, tiende a lograr un papel más activo y comprometedor en la adquisición de sus conocimientos y actitudes en el proceso de enseñanza-aprendizaje del Inglés, aspecto que en este artículo se debate para lograr la educación ambiental de forma investigativa, colaborativa y transformadora.

Palabras clave: método proyecto; educación ambiental

\section{ABSTRACT}

The project based method in the formation of university students tends to have a more active and compromising role in the acquisition of their knowledge and attitudes in the teaching-learning process of English, a topic that is debated in this article to achieve an environmental education focused on research, cooperation and transformation.

Keywords: method based on projects; environmental education

Recibido: septiembre de 2016 Aprobado: noviembre de 2016

\section{Introducción}

La globalización que hoy afecta al mundo ha significado también la generalización de múltiples problemas ambientales. El paradigma de la modernidad que suponía un ecosistema planetario inagotable, con fe ciega en la felicidad de la humanidad, a partir del desarrollo tecnológico y el sometimiento de la naturaleza, ha llegado a ser cuestionado ante el deterioro ambiental, tanto físico como social y el incremento de los llamados problemas globales, tales como la pérdida de la biodiversidad, los deficientes niveles de alimentación, el deterioro de la capa de ozono y sus consecuencias, la pobreza, el desempleo, el incremento de los precios de los alimentos a nivel mundial, los efectos del cambio climático, entre otros. Cuba sufre los efectos negativos de esta crisis ambiental mundial, y la Educación Ambiental se constituye en una necesidad social reconocida por los líderes, instituciones y organizaciones del gobierno y la sociedad civil cubana como mecanismo para mitigar dichos efectos.

La Educación Ambiental (EA) reviste gran importancia para el estado cubano, lo cual está refrendado en la Constitución de la República, en los lineamientos de la política económica del Partido Comunista de Cuba (PCC) y en la Ley 81/1997 conocida como Ley del Medio Ambiente de la República de Cuba. Para el logro interdisciplinario de este proceso educativo, esta ley debe ser de estricto cumplimiento en empresas, organizaciones, instituciones, universidades, entre otras entidades.

La Educación Ambiental plantea a la Universidad, como institución social encargada de la formación de profesionales, la necesidad de fomentar conocimientos, habilidades y valores, orientados a su comprensión y mejoramiento, a través del desarrollo de un proceso de Educación Ambiental acorde con las aspiraciones de la sociedad cubana actual, es por ello que en este artículo, se dirige la atención a una de las vías que puede favorecer la educación ambiental en la formación integral de los estudiantes universitarios: el método proyectos.

\section{Desarrollo}


Al realizar una consulta bibliográfica sobre la Educación Ambiental a nivel internacional y nacional se pudo comprobar que varios autores coincidían con que la Educación Ambiental es un proceso permanente, continuo, sistemático dirigido a la formación integral de la personalidad en su relación armónica con el entorno natural, social, económico y cultural. Valdés (1996), Camacho (1997), Novo (1998), Feijoo (2000), Bueno (2000), McPherson (2004), Fundora (2007), Mateo (2010), Santos (2010), Hernández y Pablos (2010), Iglesias (2011), Hernández, (2012), Rodríguez (2012), entre otros. Sin embargo, a pesar de las significativas aportaciones de estos autores a la teoría de la Educación Ambiental, todavía no se brindan vías en virtud de una Educación Ambiental desde la comunicación que conduzca a que los estudiantes investiguen, colaboren, reflexionen críticamente y transformen sus actitudes en la universidad, específicamente en la clase de Idiomas de las facultades de Cultura Física.

En obras como las referidas por Mateo (2010), Santos (2010), Hernández y Pablos (2010) se hace mención a la Educación Ambiental en su arista ético social, pero, a pesar de ser esta una tendencia más cercana a la formación integral del estudiante universitario, no se ofrecen vías para desarrollar la Educación Ambiental en el plano curricular, desde la teoría hasta la práctica en las universidades cubanas.

La Educación Ambiental en la enseñanza del Inglés ha sido abordada por Valladares (2009), Escobar (2010), Snezana y Veselinovska (2011), Poltavtchenko y Stoller (2012) y Pérez (2013), entre otros, debido a que en sus obras estos autores han propuesto actividades relacionadas con el medio ambiente; sin embargo, el alcance de estas actividades ha quedado en el plano cognitivo, con escasas oportunidades para que los estudiantes puedan investigar sobre el tema ambiental y colaborar en estas investigaciones.

No obstante, el tema ambiental ha provocado una creciente producción de medios para la enseñanza de lenguas extranjeras. Evidencia de ello es el número de libros de texto y videos que abordan temas medioambientales como marco para el estudio y aprendizaje del Inglés como lengua extranjera (Rabley (1989), Derwing y Cameron, (1991-1993), Martin (1991), y Stempleski (1993) los cuales se han centrado en la dimensión naturalista de la Educación Ambiental y hacia los elementos globales del medio ambiente.

A partir de la sistematización de algunas obras sobre esta relación Educación Ambiental- idiomas extranjeros, el autor coincide con el criterio de que "el aprendizaje de una lengua extranjera en su función comunicativa lleva a la concientización y comprensión de los problemas que afectan al medio ambiente. A través de la enseñanza-aprendizaje del idioma que se estudia se puede interactuar en las clases y contribuir al desarrollo social con la intención de instaurar una nueva ética del desarrollo mundial, pero también con el objetivo de fomentar actitudes positivas hacia el medio más próximo. En consecuencia, la Educación Ambiental en su relación con otras ciencias y lenguas propicia los conocimientos teóricos y prácticos, los valores y las actitudes que puedan mejorar la calidad de vida de todos los habitantes respetando el equilibrio del sistema con una perspectiva de futuro." Pérez, (2008). Para lograr esta Educación Ambiental en la clase de Inglés es necesario que los estudiantes cuenten con las condiciones básicas para lograrlo, el profesor debe tener una cultura y una Educación Ambiental adecuada para irradiar con su ejemplo y su guía. Deben existir métodos y medios que permitan la interactividad, la investigación, la colaboración y la reflexión en estos estudiantes, Por otra parte la evaluación debe proponer formas cuantitativas y cualitativas para valorar el trabajo del estudiante en este proceso educativo integrador.

Uno de los aportes más cercanos a una Educación Ambiental sistemática en la clase de Inglés aparece en un forma de artículo en la Revista English Teaching Forum(1989-1939) titulado: Linking the classroom to the world: The Environment and English Foreign Language. En este artículo la autora Stempleski, (1995) plantea que existen varias razones para introducir la temática ambiental en la clase de Inglés, estas son las siguientes:

1. La situación ambiental es de carácter urgente.

2. Los temas ambientales son una abundante fuente de contenidos.

3. Los acontecimientos ambientales son parte de la vida real.

4. Los temas ambientales motivan a los aprendices.

5. Los temas ambientales pueden vincularse a todas las áreas del currículo. (interdisciplinariedad) 6 . Los temas ambientales proveen un marco propicio para integrar las habilidades lingüísticas.

7. Los temas ambientales promueven la interactividad.

8. Aprender sobre los temas ambientales produce placer y promueve curiosidad sobre temas globales y locales.

Esta obra aporta un referente teórico muy importante para los autores de este artículo, pues sirve de base para el estudio y sistematización de la Educación ambiental en el PEA del Inglés, pero insistiendo en el método proyecto como vía para la interdisciplinariedad este proceso de la forma- 
ción integral del estudiante universitario para su educación ambiental.

El método proyectos se enmarca dentro del enfoque histórico cultural, asumido como referente que vinculan a la Educación Ambiental con el PEA del Inglés, entre ellos resaltan: la teoría de la zona del Desarrollo Próximo (ZDP) y la importancia que Vygotsky le confiere a las experiencias vivenciales para el aprendizaje.

"La Zona de Desarrollo Próximo (Vygotsky) se refiere al espacio, brecha o diferencia entre las habilidades que ya posee el alumno y lo que puede llegar a aprender a través de la guía o apoyo que le puede proporcionar el maestro o un par más competente." Vygotsky, (1988 p. 43). Según Vygotsky, la enseñanza debe partir del nivel de desarrollo real, lo que permite al sujeto avanzar en el conocimiento y desarrollarse progresivamente; por este motivo, enseñar supone crear Zonas de Desarrollo Próximo adelantándose siempre al desarrollo. La ZDP es la mejor alternativa para la integración educativa. Convirtiendo así una experiencia individual, en un proceso social. El estudiante sostenido por la ayuda del profesor, de un compañero o herramientas como una multimedia, recorre esa zona construyendo conocimiento, dejando establecidos nuevos niveles de desarrollo real y potencial, que delimitan una nueva Zona de Desarrollo Próximo (ZDP). Con la ayuda del docente, en la ZDP, los estudiantes pueden lograr ciertos aprendizajes sobre todo el relacionado con la Educación Ambiental que antes solamente eran potenciales. Esto permite que se consiga no solamente un nuevo nivel de desarrollo real, sino también, y, lo más importante, un nuevo nivel de desarrollo potencial que posibilita una nueva y más avanzada ZDP, en la que antes no se lograba realizar actividades ni solos ni acompañados.

Según el autor es muy viable la aplicación de la ZDP en la clase de Inglés, porque esta zona es el espacio en que gracias a la interacción y la ayuda de otros, el estudiante de Cultura Física puede trabajar y resolver una situación comunicativa o realizar una tarea interactiva de una manera y con un nivel que no sería capaz de tener individualmente.

Respecto a las experiencias vivenciales para el aprendizaje, Vygotsky plantea que: "es en la vivencia en donde se articula el medio en su relación con la persona, la forma en que ella vive y además se manifiestan las particularidades del desarrollo de su propio yo Si se tuviera que formular una tesis formal, un tanto generalizada, sería correcto decir que el medio determina el desarrollo del sujeto a través de la vivencia de dicho medio. Así pues, lo más esencial es renunciar a los índices absolutos del medio." Vygotsky, (1988 p. 76) Como otro aporte desde la concepción de Vygotsky de asumir el desarrollo de la personalidad como una unidad indisoluble entre los factores biológicos y sociales se expresa que "El aprendizaje vivencial ocurre cuando el individuo se involucra y participa en cierta actividad, reflexiona sobre lo acontecido en forma crítica, extrae importantes conclusiones desde el análisis introspectivo e incorpora lo aprendido a través de un cambio en la forma de pensar o comportarse" Morales, (2015)

A la luz de este enfoque histórico cultural se plantean algunas concepciones de aprendizaje que por su implicación en el desarrollo integral de la personalidad se considera de gran importancia destacar en esta tesis, una es la referente al aprendizaje desarrollador.

"Un aprendizaje desarrollador es aquel que garantiza en el individuo la apropiación activa y creadora de la cultura, propiciando el desarrollo de su auto perfeccionamiento constante, de su autonomía y autodeterminación, en íntima conexión con los necesarios procesos de socialización, compromiso y responsabilidad social." Colomé, (2012 s/p) La Educación Ambiental con una postura ético social posibilita este aprendizaje desarrollador, solo que con su actual carácter reduccionista hacia las Ciencias Naturales, este tema se mantiene restringido hacia la práctica del idioma Inglés en sus objetivos comunicativos.

Una Educación Ambiental no sólo desde los contenidos como se percibe en los programas y cursos de Inglés para la carrera de Cultura Física sino desde el redimensionamiento de todas las categorías didácticas, permitiría que el estudiante fuera aún más protagonista de su aprendizaje y gradualmente avanzar hacia niveles superiores de su aprendizaje como así se plantea en la teoría del aprendizaje desarrollador, pues este "debe potenciar en los/las estudiantes la apropiación activa y creadora de la cultura. Representa aquella manera de aprender y de implicarse en el propio aprendizaje que garantiza el tránsito del control del proceso por parte del/la docente al control por parte de los/las aprendices, y, por ende, conduce al desarrollo de actitudes, motivaciones y herramientas necesarias para el dominio de aquello que llamamos aprender a aprender, y aprender a crecer de manera permanente." Castellanos, (2005, p.3)

Los autores declaran que la Educación Ambiental por su intencionalidad de formar una personalidad que desarrolle sus conocimientos, habilidades, actitudes y valores respecto al medio ambiente en todas sus dimensiones se convierte en una vía, un vehículo para lograr la competencia com- 
portamental que es sin dudas una de las áreas más importantes de la competencia comunicativa como objetivo esencial de una clase de lenguas extranjeras.

Otro elemento didáctico que ha sido concebido en los planes de estudio, específicamente en el plan de estudio $D$ con el curso de Inglés At your Pace y se considera el más importante para lograr esta Educación Ambiental a que se aspira, es el empleo del método de proyectos.

El método de proyectos "es una metodología que va desde la identificación de un problema hasta la solución del mismo, pasando por etapas que incluyen la búsqueda de información, el diseño y elaboración de prototipos, ensayos, construcción, comunicación. Es ésta una técnica que ha tenido gran desarrollo y aplicación en diferentes contextos, principalmente, relacionados con ámbitos técnico - tecnológicos."(http://integracionmediatecsog. blogspot.com/2009/06/ metodo-proyectos-aprendizaje-basado-en.html. consultado el 15 de abril de 2015)

Algunos estudiosos del tema (Kilpatrick (1998), Frey (1982), Reisch (1990) y Álvarez (2012) plantean que este orienta los procedimientos y les confiere una motivación, una actividad intencional, un plan de trabajo, un conjunto de tareas que tienden a una adaptación individual y social, pero emprendidas voluntariamente por el estudiante o por el grupo. Estas actividades colocan al estudiante dentro de una micro realidad, la de los problemas vivenciales.

A partir de los criterios de Dewey \& Kilpatrick (1935); Aguayo (1943), Freire (1970); Schultz, (1973); Rodríguez (1975); Freinet (1976); Stenhouse (1987); Makarenko (1996), la práctica educativa debe ser diferente a la de otros siglos anteriores, el conocimiento científico debe imponerse y las clases reproductivas deben cambiarse por enseñanzas que utilicen proyectos basados en el diálogo, la participación, la cooperación entre los integrantes de esta práctica donde la didáctica esté centrada en los estudiantes.

El método de proyectos se aplica en el mundo desde el siglo XVII en Francia y los que promovían este método lo usaban como un medio para un objetivo práctico, vinculado con una solución a corto plazo como construir cañerías, acueductos, calles, subestimando su valor pedagógico. Este método llegó a Estados Unidos donde se le atribuyó, en su concepción pragmática, la posibilidad de que los estudiantes pudieran crear y hasta vender el resultado de sus proyectos. No fue hasta la aparición de la Reforma Pedagógica que este método llegó a las universidades norteamericanas.

Es importante mencionar en el desarrollo de este método, el surgimiento del proyecto Warisata, una de las experiencias latinoamericanas de proyecto que trabajó pedagógicamente desde la perspectiva de la investigación, acción, participación, colaboración y transformación de las realidades concretas. Boller (2004); Mora (2006). Según esta experiencia los componentes del método de proyectos son: iniciativa, discusión, planificación, desarrollo, culminación, formalización y la evaluación que se mantiene presente en todos los componentes "Cuando se utiliza el método de proyectos como estrategia, los estudiantes estimulan sus habilidades más fuertes y desarrollan algunas nuevas. Se motiva en ellos el amor por el aprendizaje, un sentimiento de responsabilidad y esfuerzo y un entendimiento del rol tan importante que tienen en sus comunidades." (Blumenfeld. 1991, p. 56)

Este método beneficiaría significativamente a la Educación Ambiental de los estudiantes en una clase de Inglés, pues mediante este tienen la posibilidad de investigar sobre los temas ambientales que más le interesen, se comprometen y se sensibilizan con ellos. Con el empleo de este método los estudiantes pueden contar con la ayuda de los más aventajados para fortalecer el trabajo colaborativo, pueden utilizar las tecnologías de la información como páginas web o multimedia y cámaras digitales para llevar fotos que muestren evidencias.

Además de investigar, este estudiante mediante el método proyecto debe convertirse en un educador ambientalista eficaz, por eso los autores consideran como referente a tener en cuenta las recomendaciones de Bueno, (1998), que se traducen en:

1- Valorar el papel que desempeñan los distintos conocimientos y las experiencias en el fomento de las conductas deseadas por la Educación Ambiental.

2- Fomentar la capacidad de transferencia de actitudes, habilidades y valores ambientales en sus educandos, así como la toma de decisiones para preservar el equilibrio entre la calidad de vida humana y el entorno.

3- Elegir las metodologías adecuadas para conseguir determinados objetivos cognitivos y afectivos de la Educación Ambiental.

4-Infundir y evaluar los contenidos y métodos elegidos, así como la eficacia de la estrategia ambiental aplicada.

5-Emplear los conocimientos y técnicas necesa- 
rias para reconocer e investigar problemas ambientales.

6- Dominar la legislación vigente sobre la protección del medio ambiente.

El cumplimiento de los aspectos antes mencionados se hace posible en el desarrollo de la Educación Ambiental en la asignatura Inglés mediante el método de proyectos y debe ser entendida como un proceso didáctico. La estructuración de este proceso se fundamenta en una didáctica desarrolladora e integradora, que sistematiza los aportes desde el punto de vista filosófico a partir de la teoría del conocimiento (Lenin, 1959), así como la significatividad del mundo al asumir posiciones dadas. (Marx 1973).

Respecto al método de proyectos es de obligada consulta la tesis de Álvarez (2012) titulada: "Un modelo didáctico, centrado en el método de proyectos para contribuir al desarrollo de la autonomía en el aprendizaje del Inglés desde la Práctica Integral de la Lengua inglesa en la formación inicial de profesores de la carrera de lenguas extranjeras." Según este autor "el método de proyectos emerge de una visión de la educación en la cual los estudiantes toman una mayor responsabilidad de su propio aprendizaje y aplican, en proyectos reales, las estrategias y conocimientos adquiridos en el aula; busca enfrentar los estudiantes a situaciones que los lleven a comprender y aplicar aquello que aprenden como una herramienta para resolver problemas o proponer mejoras en los diferentes contextos sociales donde se desenvuelven. Cuando se utiliza el método de proyectos, se estimula en los estudiantes las habilidades ya formadas y se desarrollan otras nuevas, se motiva en ellos el amor por el aprendizaje, un sentimiento de responsabilidad y esfuerzo y un entendimiento del papel que juegan en la solución de problemas de aprendizaje y sociales. Además, se tienen en cuenta sus necesidades y motivaciones, fortaleciéndose el plano afectivo en el aprendizaje." (Álvarez. 2012 p. 34)

A diferencia de esta tesis, el autor de la presente investigación asume este método para el desarrollo de la Educación Ambiental en el proceso de enseñanza-aprendizaje del Inglés teniendo en consideración que mediante este método el estudiante puede transformar sus actitudes desde la práctica comunicativa, colaborativa e investigativa.

Es importante aclarar en esta investigación que la Educación Ambiental que se pretende desarrollar en esta investigación, mediante el método de proyectos, fortalece los objetivos formativos de la educación y de la enseñanza del Inglés como lengua extranjera, como también ofrece vías para su ejecución práctica desde la adquisición de conocimientos hasta el cambio en sus actitudes.

Las actividades que se emplean en este método proyecto son principalmente tareas interactivas dentro y fuera de la clase. Estas se enfocan desde una perspectiva: 1) Crítica, 2) Investigativa, 3) Colaborativa y transformadora. De esta forma, los estudiantes son capaces de desarrollar análisis críticos de los temas que se orienten en las tareas de aprendizaje. Mediante el método de proyectos se realiza un estudio investigativo sobre los problemas ambientales que se asignen en la clase práctica de Inglés y el trabajo en equipo fortalece el aprendizaje desarrollador y formativo del estudiante y con ello la relación personal y profesional en el colectivo.

Los medios constituyen el soporte material del método; en las actividades de Educación Ambiental a partir del método de proyectos, el medio por excelencia es el medio ambiente en todas sus dimensiones, convirtiéndose este en elemento esencial del proceso enseñanza - aprendizaje en esta actividad pues en dependencia de sus características, se reorientarán los objetivos y demás componentes del proceso. En este caso la selección del lugar donde se realizarán las actividades mediante el método de proyectos es fundamental pues debe cumplir con los objetivos que se proponen.

Otros medios muy importantes para el proceso de Educación Ambiental son: mapas verdes, mapas de la localidad, termómetros, cintas métricas, dispositivos para evaluar la calidad del agua (donados por el Programa Internacional para el Monitoreo del Agua), también son muy útiles las cámaras digitales, los reproductores de video, la computadora y la multimedia Fernavisión sobre medio ambiente, deportes y la enseñanza del Inglés donde los estudiantes encontrarán información sobre este tema, además de los parámetros para convertirse en educadores ambientales en sus futuras prácticas educativas y en la comunidad.

\section{Conclusiones}

Las etapas por las que ha transitado la evolución de la Educación Ambiental y el tratamiento dado al ambiente parten de una tendencia conservacionista que no considera el impacto de la actividad social y las necesidades humanas en el ambiente. Después se experimenta un período de conciencia ecologista que transita a posiciones más ambientalistas sobre la base del desarrollo, hasta llegar a la Educación Ambiental como un proceso educativo continuo y permanente; este, en el contexto educativo, propicia el protagonismo de los estudiantes en el aprendizaje y la Educación 
Ambiental, desde lo natural, social, y hacia el medio ambiente construido.

El desarrollo de acciones relacionadas con la Educación Ambiental en el proceso de enseñanza-aprendizaje del Inglés ha sido ecologista, contemplativo, priorizando la competencia lingüística según tendencias de la enseñanza de lenguas extranjeras de la época, es por ello que el método proyectos tiende a lograr en los estudiantes un valor más activo y comprometedor en los conocimientos y actitudes en el proceso de enseñanza-aprendizaje del Inglés y de esta forma sea más crítica, investigativa, colaborativa y transformadora.

\section{Referencias bibliográficas}

1. Acosta Padrón R \& A. Hernández J (2008) Didáctica Interactiva de la Comunicación,Pinar del Río, Cuba, pp. 5-6

2. Acosta Paula. A. C. (2011).Modelo didáctico, basado en un enfoque profesional - interdisciplinar, para contribuir a la educación energética en la formación inicial de profesores de ciencias exactas. Pinar del Río. 120h. Tesis presentada (en opción al grado científico de Doctor en Ciencias Pedagógicas). Universidad de Ciencias Pedagógicas Rafael María de Mendive

3. Aguayo Alfredo M (1943) Didáctica de la Escuela Nueva, Imprenta cultural, La Habana, p. 58. 4. Álvarez Legrá E. (2012) Un modelo didáctico, centrado en el método de proyecto para contribuir al desarrollo de la autonomía en el aprendizaje del inglés desde la PILI, en la formación inicial de profesores de la carrera de lenguas extranjeras. Tesis en opción al grado científico de Doctor en Ciencias. pp. 67

5. Antich de León, 30 R.; C. D. Gandarias \& S. E. López. (1986). Metodología de la enseñanza de lenguas extranjeras. La Habana: Pueblo y Educación, p. 60.

6 Blumenfeld, P. C., Soloway, E., Marx, R. W., Krajcik, J. S., Guzdial, M., \& Palincsar, A. (1991). Motivating project-based learning: Sustaining the doing, supporting the learning. Educational Psychologist, 26 (3 \& 4).

7. Bosque, R., Merino, T. y Fundora, J. (2007). Educación Ambiental para el Desarrollo Sostenible. En Maestría en Ciencias de la Educación. Módulo III. Cuarta Parte. Mención en Educación Preuniversitaria. (pp. 31-54). La Habana: Pueblo y Educación.

8. Bueno, S, E F. (2000). Maestría en didáctica de la geografía. Soporte magnético. La Habana: ISPEJV.

9. Castellanos D. (2005) Estrategias para promover el aprendizaje desarrollador en el contexto escolar. Pedagogía 2005 Educación Cubana. pp. 18 10. Colomé Medina J. A. (2012) Concepciones actuales acerca del aprendizaje Desarrollador y Formativo. Monografías. ISP Juan Marinello.

11. De Armas Ramírez, Nerelys et al. (2004): Caracterización y diseño de los resultados científicos como aportes de la investigación educativa, Curso 85, Evento Internacional 27.Pedagogía 2003, La Habana

12. Fiallo, J. (1996) Las relaciones intermaterias: una vía para incrementar la calidad de la educación. La Habana: Pueblo y Educación.

13. Frey, K. (1982). El método de proyectos. Weinheim/ Basel: Beltz.

14. Kilpatrick, W. Disponible en http://es.wikipedia.org/wiki/William_Heard_Kilpatrick. Consultado el 12 de marzo de $201 \overline{4}$.

15. Ley 81 del Medio Ambiente. (1997). La Habana: Gaceta oficial de la República de Cuba. Edición extraordinaria. Año XCV

16. Mc Pherson Sayú, M., Hernández Herrera, P., Franco Suárez, M., Díaz Castillo, R., Bayón Martínez, P. y Amador Lorenzo, E. L. (2004). La Educación Ambiental en la Formación de los Docentes. La Habana: Pueblo y Educación.

17. Morales Delegado J. C. (2001) Propuesta metodológica para la educación ambiental en Ciencias naturales 5 to grado. Tesis en opción al título académico de Máster en "Didáctica de la geografía ISP Enrique José Varona. La Habana

18.Morales Fuentes, C. M. (2011).Estrategia para la superación profesional en educación energética de los docentes de preuniversitario. Camagüey .120 h. Tesis presentada (en opción al título de Doctor en Ciencias Pedagógicas. Universidad de Ciencias Pedagógicas de José Martí.

19. Pérez Mauri. M. C. (2008) La dimensión ambiental en la enseñanza del idioma alemán. Monografías. Universidad de Matanzas. Camilo Cienfuegos

20. Pérez Morales J. I. (2013) La educación ambiental desde el inglés con fines académicos. Universidad Central "Marta Abreu" de Las Villas. Cuba. CD de Memorias del 9no Congreso Internacional de Educación Superior. Universidad 2014.

21. Valcárcel Izquierdo N. (1998). Estrategia interdisciplinaria de superación para profesores de ciencias de la enseñanza media. Resumen de Tesis presentado en opción al Grado Científico de Doctor en Ciencias pedagógicas, La Habana.

22. Valdés Valdés O. (1996). La Educación Ambiental en el Proceso Docente Educativo en las Montañas de Cuba. La Habana: Resumen de tesis presentada (en opción al grado científico de Doctor en Ciencias Pedagógicas).

23. Valladares Fuente F. (2009) Sistema de actividades de aprendizaje para contribuir a la educación ambiental desde el Inglés. Tesis en opción al grado académico de Máster. Universidad de Ciencias Pedagógicas "Rafael María de Mendive" 
Pinar del Río.

24. Vygotsky, L. (1988). "Cap. IV: Internalización de las funciones psicológicas superiores", y "Cap. VI: Interacción entre aprendizaje y desarrollo", en: El desarrollo de los procesos psicológicos superiores, Crítica, Grijalbo, México, pp. 87-94 y 123140. 